\title{
A Review of Target Population Estimates and Implied Infant Mortality Rates from National Immunization Programmes During 2000-2010
}

\author{
David W Brown*, Anthony H Burton, Marta Gacic-Dobo and Rouslan Karimov
}

United Nations Children's Fund (UNICEF), New York, New York (DWB, RK), World Health Organization (WHO), Geneva, Switzerland (AHB, MGD)

\begin{abstract}
Because complete vital registration data often do not exist, immunization programme managers must estimate the number of children in the target population for computing immunization coverage, an important measure used to monitor immunization programme performance. This report presents information on the estimated number of live births and of surviving infants for the years 2000 - 2010 received by the World Health Organization (WHO) and United Nations Children's Fund (UNICEF) from national immunization programmes (data received as of July 2011) and the implied infant mortality rate (IMR) according to national immunization programme estimates. This information is compared to external sources. The results highlight potential challenges confronted by immunization programme managers in reporting target population estimates at the national level and shows that the estimated number of live births and surviving infants submitted to WHO and UNICEF in the Joint Reporting Form for Immunization are inconsistent with estimates from the United Nations Population Division and implied infant mortality levels are inconsistent with estimates from external sources.
\end{abstract}

Keywords: Immunization, target population, infant mortality rate.

\section{INTRODUCTION}

As a matter of practice, immunization programme managers routinely monitor the number of children that are immunized. In addition, they must also estimate the target population size when planning services, managing stocks and supplies and targeting interventions. An estimate of the target population is also necessary for computing immunization coverage, an important measure used to monitor immunization programme performance.

Because complete vital registration, the most reliable source for such data, does not exist in the majority of the low- and middle-income countries [1,2], immunization programme managers must estimate the number of children in the target population (e.g., surviving infants) based on counts or estimates by local programme staff or health workers or rely on population projections from the latest census data [3]. Population projections are complex computations [4] that often make use of estimates of fertility and mortality in conjunction with the number of women of reproductive age to obtain estimates of the number of births. Estimates of infant mortality are applied to estimated numbers of births to obtain an estimate of the number of children who survive to their first birthday. At each step of this process there is uncertainty in the parameter estimates used [3], and this uncertainty increases the further one is from the last census. Changing

*Address correspondence to this author at the United Nations Children's Fund, Three UN Plaza, New York, New York, USA 10017;

Tel: +1.212.303.79.88; E-mail: dbrown@unicef.org fertility, mortality and/or migratory patterns over time create further challenges for the immunization programme manager in this regard.

In this report we present information on the estimated number of live births and of surviving infants for the years 2000 - 2010 received by the World Health Organization (WHO) and United Nations Children's Fund (UNICEF) from national immunization programmes (data received as of July 2011), and we compute (where possible) the implied infant mortality rate (IMR) according to national immunization programme estimates of the number of births and surviving infants. We compare this information to the most recent IMR estimates from the United Nations Interagency Group on Child Mortality Estimation (IGME) [5] and the United Nations Population Division (UNPD).

\section{METHODS}

Since 1998, WHO and UNICEF have jointly collected national-level data on the incidence of selected vaccinepreventable diseases, immunization coverage, recommended immunization schedules, vaccine supply and other information on the structure, policies and performance of national immunization systems through the WHO/UNICEF Joint Reporting Form on Immunization (JRF) [6]. Since 2000, more than $95 \%$ of WHO Member States have reported annually. As part of this annual exercise, national authorities report the estimated number of children vaccinated according to administrative data (i.e., reports from health service providers), the number of children in the national target population for each antigen in the national immunization schedule. 
The appropriate target population for vaccines administered at birth (eg., Bacille Calmette-Guérin or BCG) is the estimated number of live births in the country. For other antigens, the target population most often used for computing coverage is the number of surviving infants. (NB. Some countries use live births as the official denominator for coverage levels for diphtheria and tetanus toxoid with pertussis [or DTP] containing vaccine, polio vaccine, hepatitis B and Hib containing vaccines, and yellow fever vaccine.)

Using databases maintained by the WHO and UNICEF, we abstracted the estimated number of live births and surviving infants reported by national authorities in the JRF for the period 2000 - 2010, and we compared these data with estimates reported by the UNPD World Population Prospects, 2010 edition [7]. Country level data reported by UNPD are not available for areas with $<100,000$ inhabitants.

From the JRF data we computed an implied IMR, expressed per 1000 live births, for each country where possible based on the national estimated number of births and surviving infants using the standard formula:

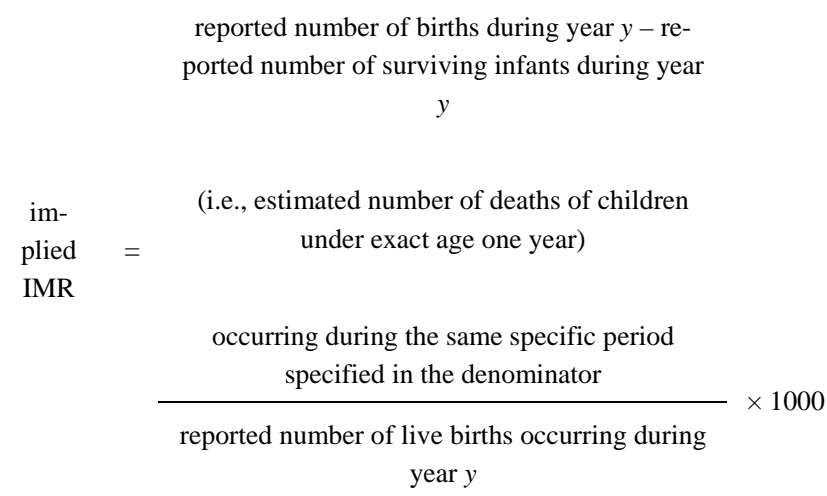

Although some infant deaths occurring during a calendar year (y) are to children born in the preceding year $(y-1)$ and some children born during a calendar year (y) who die in infancy do so in the following year $(y+1)$, the number of births and deaths in a given year (y) will approximate those in neighbouring years assuming the number of births and deaths are not changing rapidly from year-to-year.

We then compared the implied IMR from the nationally reported data with estimated IMR as reported by IGME [5] for each country during 2000-2010 by computing the percent difference using the standard formula:

$\begin{gathered}\text { Percent } \\ \text { difference }\end{gathered}=\frac{\text { absolute value of ( IMR_computed }-}{\text { average of (implied IMR and IMR_IGME) }} \times 100 \%$

A similar comparison was made using IMR estimates from UNPD data.

\section{RESULTS}

During the period 2000 - 2010, there were a total of 2126 possible reporting events (194 countries or territories reporting 11 years; Timor-Leste became Member State in 2002 and data reporting started in 2002; data reporting for Montenegro started in 2006) to WHO and UNICEF as part of the annual immunization data collection exercise. Countries reported information on the number of live births in 1614 (76\%) possible reporting events. Non-reporting of the number of live births by countries where BCG vaccine is not included in the national immunization schedule occurred in 31 countries ${ }^{1}$ for the period 2000 - 2010 (341 possible reporting events); eight countries reported the number of live births in some years but not others, despite the absence of BCG in the national schedule during 2000 - 2010 (representing 47 possible reporting events) ${ }^{2}$; and the Czech Republic removed BCG from the national schedule beginning in 2010 (1 reporting event). Countries reported the number of surviving infants in 1766 (83\%) reporting events.

One hundred thirty-two countries reported the number of live births for the target population for all antigens in 717 reporting events; eleven countries (Bolivia, Brazil, Colombia, Ecuador, Honduras, India, Jamaica, Maldives, Myanmar, Nepal, El Salvador) reported the number of live births for the target population of all antigens in each of the 11 reporting years reviewed here. For these 717 reporting events we were not able to compute an IMR from the nationally reported JRF data. An IMR was also not computed for 530 reporting events where countries did not report data on either live births or surviving infants. In 146 reporting events across 49 countries, the nationally reported estimate of the number of live births in the JRF was less than the number of surviving infants, resulting in an implausible negative IMR value.

Of 733 reporting events from 125 countries $^{3}$ where nationally reported data on live births and surviving infants were available to compute a (non-negative) IMR (referred to as the national IMR here), the national IMR value differed from IGME estimates by $</=10 \%$-points (absolute difference) in 158 (21\%) reporting events (Fig. 1). The (absolute) difference between the national IMR and IGME estimate was 11-25\%-points in 151 (21\%) reporting events and was $>25 \%$-points in 424 (58\%) reporting events. In 149 reporting events the national IMR differed from IGME estimates by more than $100 \%$-points suggesting possible reporting prob-

${ }^{1}$ Andorra, Antigua \& Barbuda, Australia, Austria, Belgium, Bahamas, Barbados, Canada, Switzerland, Cyprus, Germany, Denmark, Spain, Finland, France, United Kingdom, Greece, Grenada, Iceland, Israel, Italy, Kuwait, Lebanon, Luxembourg, Monaco, Netherlands, New Zealand, Saudi Arabia, San Marino, Sweden, United States

2Bahrain, reported in 2000; Japan, reported during 2000-2006; Jordan, 2000-2001; Malta, 2000-01,2003-04; Palau, 2000-07; Suriname, 2000-02, 2005-2010; Slovenia, 2005-2010; Trinidad \& Tobago, 2001-2010

${ }^{3}$ Afghanistan, Albania, Angola, Azerbaijan, Bangladesh, Barbados, Belarus, Belize, Benin, Bhutan, Bosnia \& Herzegovina, Botswana, Brunei, Bulgaria, Burkina Faso, Burundi, Cameroon, Cape Verde, Central African Republic, Chad, Chile, China, Comoros, Congo, Congo DR, Cook Islands, Costa Rica, Cote d Ivoire, Croatia, Cuba, Czech Republic, Djibouti, Dominica, Egypt, Equatorial Guinea, Eritrea, Estonia, Ethiopia, Fiji, Gabon, Gambia, Georgia, Ghana, Guinea, Guinea-Bissau, Haiti, Hungary, Indonesia, Iran, Iraq, Japan, Jordan, Kazakhstan, Kenya, Kiribati, Korea DPR, Korea Rep, Kyrgyzstan, Lao PDR, Latvia, Lesotho, Liberia, Libya, Lithuania, Macedonia, Madagascar, Malawi, Malaysia, Mali, Malta, Marshall Islands, Mauritania, Mexico, Moldova, Mongolia, Montenegro, Morocco, Mozambique, Namibia, Nauru, Nicaragua, Niger, Nigeria, Oman, Pakistan, Papua New Guinea, Philippines, Poland, Portugal, Qatar, Russian Federation, Rwanda, Saint Kitts \& Nevis, Saint Lucia, Samoa, Sao Tome \& Principe, Senegal, Serbia, Seychelles, Sierra Leone, Slovakia, Somalia, South Africa, Sri Lanka, St Vincent \& the Grenadines, Sudan, Swaziland, Tajikistan, Tanzania, Timor Leste, Togo, Tonga, Tunisia, Turkey, Turkmenistan, Tuvalu, Uganda, Ukraine, Uruguay, Uzbekistan, Vanuatu, Vietnam, Yemen, Zambia, Zimbabwe 


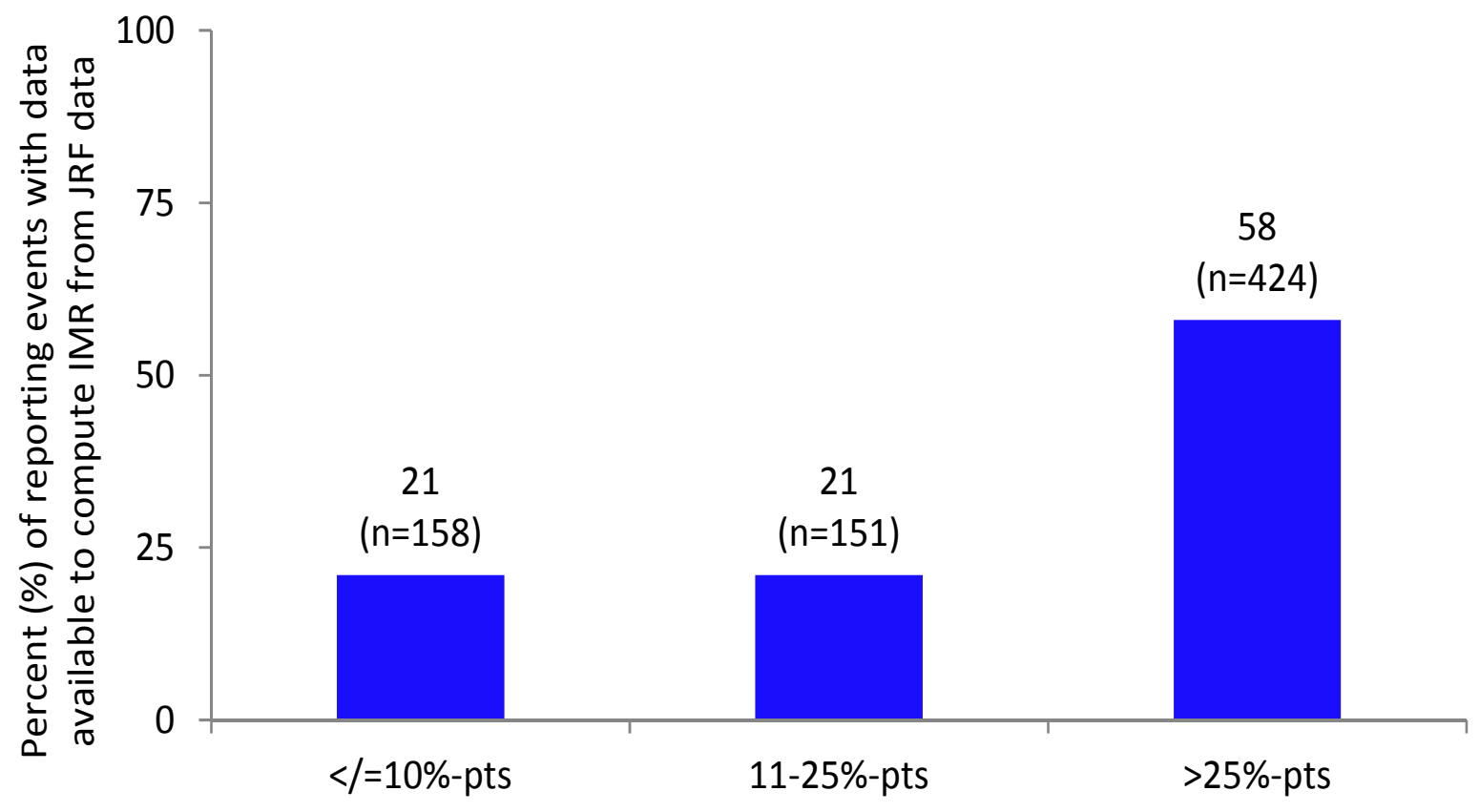

\section{Absolute difference between national IMR computed from JRF data and IGME estimated IMR}

Fig. (1). Absolute difference between national infant mortality rate computed from JRF data and the IMR based on IGME estimates and UNPD estimates across 125 countries (733 reporting events) for the period 2000-2010.

lems in the target population estimates reported on the Joint Reporting Form. Similar results were observed for IMR comparisons with UNPD estimates (Fig. 1; an IMR comparison was not made for 25 of 733 reporting events from small island states where UNPD population data are $<100,000$ persons).

A total of 570 (78\%) reporting events with a (nonnegative) national IMR were from developing or least developed countries (according to World Bank classification [8] (Table. 1). In these countries, the national IMR differed from IGME estimates by $</=10 \%$-points in $145(25 \%)$ reporting events; 11-25\%-points in 137 (24\%) reporting events; and $>25 \%$-points in 288 (51\%) reporting events. Similar findings were observed among the 373 reporting events with a nonnegative IMR value from African countries (IMR percent difference $</=10 \%$ points in $106(28 \%)$ events; $11-25 \%$ points in 105 (28\%) events; and >25\%-points in 162 (43\%) reporting events).

Plots of these data over time are shown below for select countries (available at https://sites.google.com/site/impliedIMRproject). Consistent with the summary above, nationally reported data are available for some but not all countries.

\section{DISCUSSION}

This review highlights potential challenges confronted by immunization programme managers in reporting target population estimates at the national level. For many countries, the estimated number of live births and surviving infants submitted to WHO and UNICEF in the Joint Reporting Form for
Immunization were inconsistent with estimates from the United Nations Population Division (the number of live births was within $10 \%$ of the UNPD estimate of live births in 890 [55\%] events; the number of surviving infants was within 10\% of the UNPD estimate in 1011 [57\%] events), and implied infant mortality levels from JRF data were inconsistent with estimates from IGME, UNPD, or both. Differences between the implied IMR and estimates from other sources are not unexpected though we were somewhat surprised to observe nearly $60 \%$ of reporting events having a difference of more than 25\%-points when compared to the UN estimates. While differences may exist with regards to estimation methods, for example how agencies and governments might define or classify a live birth [9], such differences would be unlikely to lead to large (>25\%-point) relative differences in IMR between the sources examined here. Data suggesting imnlausible negative IMR values were also observed, and such cases may simply suggest data entry errors, although for 19 countries the error was repeated over multiple ( $>2$ ) years.

In one third of the possible reporting events during 20002010 countries reported the estimated number of live births as the target population for all antigens. The use of live births as the target population for all antigens is not in-andof-itself problematic particularly if infant, and especially neonatal, mortality levels are low. Of course, the practical implication for performance monitoring is that estimated immunization coverage would be lower than it should be by assuming no infant mortality for say the proportion of infants immunized with three doses of polio vaccine. In some coun- 
Table 1. Number of Possible Reporting Events to the WHO/UNICEF JOINT Reporting Form on Immunization During 2000-2010, Number of Events with a Computed IMR from Nationally Reported Data by Category of Agreement with Estimates from the UN IGME by Regional Classification

\begin{tabular}{|c|c|c|c|c|c|}
\hline \multirow[t]{2}{*}{ Regional Classification } & \multirow{2}{*}{$\begin{array}{l}\text { Number of Possible } \\
\text { Reporting Events, } \\
\text { 2000-2010 (N=2126) }\end{array}$} & \multirow{2}{*}{$\begin{array}{l}\text { Number of Reporting } \\
\text { Events with Computed } \\
\text { IMR from JRF data } \\
\text { (N=733) }\end{array}$} & \multicolumn{3}{|c|}{$\begin{array}{l}\text { Number of Reporting Events by Percent Difference } \\
\text { between National IMR Computed from JRF Data } \\
\text { and UN-IGME IMR Estimates }\end{array}$} \\
\hline & & & $</=10 \%$ pts & $11-25 \% p t s$ & $>25 \%$ pts \\
\hline \multicolumn{6}{|l|}{ MDG Region } \\
\hline Latin American and Caribbean & 363 & 34 & 5 & 2 & 27 \\
\hline Caucasus and Central Asia & 88 & 61 & 3 & 4 & 54 \\
\hline East Asia & 44 & 26 & 4 & 3 & 19 \\
\hline West Asia & 143 & 30 & 14 & 7 & 9 \\
\hline Southern Asia & 99 & 47 & 14 & 10 & 23 \\
\hline South East Asia & 119 & 19 & 2 & 5 & 12 \\
\hline North Africa & 55 & 33 & 4 & 9 & 20 \\
\hline Sub-Saharan Africa & 528 & 340 & 102 & 96 & 142 \\
\hline Oceania & 154 & 41 & 0 & 5 & 36 \\
\hline Developed & 533 & 102 & 10 & 10 & 82 \\
\hline \multicolumn{6}{|l|}{ WHO Regions } \\
\hline Africa & 506 & 312 & 93 & 93 & 126 \\
\hline The Americas & 385 & 34 & 5 & 2 & 27 \\
\hline Eastern Mediterranean & 242 & 116 & 39 & 25 & 52 \\
\hline Europe & 577 & 162 & 13 & 14 & 135 \\
\hline South East Asia & 119 & 32 & 2 & 5 & 25 \\
\hline Western Pacific & 297 & 77 & 6 & 12 & 59 \\
\hline \multicolumn{6}{|l|}{ UNICEF Regions } \\
\hline CEE-CIS & 225 & 119 & 6 & 7 & 106 \\
\hline East Asia and Pacific & 317 & 86 & 6 & 13 & 67 \\
\hline South Asia & 88 & 36 & 8 & 7 & 21 \\
\hline Middle East and North Africa & 231 & 94 & 27 & 21 & 46 \\
\hline East and Southern Africa & 242 & 157 & 42 & 41 & 74 \\
\hline West and Central Africa & 264 & 162 & 57 & 53 & 52 \\
\hline The Americas and Caribbean & 363 & 34 & 5 & 2 & 27 \\
\hline Industrialized & 396 & 45 & 7 & 7 & 31 \\
\hline
\end{tabular}

tries information on fertility patterns may be more robust than that for infant mortality, particularly over time, and thus this approach may be an appropriate one. It is important to note, however, that immunization programme managers should be cautious if they use estimated number of births based on fixed factors (e.g., the estimated number of births is equal to $4 \%$ of the total population) since such an approach can introduce a trend bias and ultimately impact the plausibility of immunization coverage estimates [10].

The results observed here may also reflect situations where countries systematically "back calculate" the target population using estimates of immunization coverage and the 
estimated number of children vaccinated. Unfortunately, such situations are difficult to diagnose. Such an approach can be useful in the process of triangulating target population estimates in the face of little information or untimely information (e.g., such as the case may be with very old census data), but as a matter of practice, this is sub-optimal.

Finally, we encourage immunization programme managers to visually examine the data reviewed here over time as part of their annual data reviews. Plots of the country-level data allow one to quickly observe large differences in implied IMR used by national programmes relative to estimates from other sources (e.g., Uganda at https://sites.google.com/site/impliedIMRproject/) and patterns such as fluctuating IMRs over time that are consistent with trends from other sources (e.g., Afghanistan at https://sites.google.com/site/impliedIMRproject/), stable IMRs over time that are consistent with trends from other sources but at different levels of IMR (e.g., Burundi and Burkina Faso at https://sites.google.com/site/impliedIMRproject/) and IMR levels that are inconsistent with the trends observed from other sources (e.g., Bangladesh https://sites.google.com/site/impliedIMRproject. Because national immunization coverage derived from administrative data is sensitive to the underlying estimated number of live births and the estimated IMR in a country, visualizing such patterns in IMR and the target population may be useful in further understanding observed coverage patterns. For example, one can readily see that for a given number of children vaccinated and birth cohort size, if the estimated IMR is too high, then the number of surviving infants is smaller than it should be leading to an overestimate of immunization coverage. Likewise, an underestimate of the true IMR may lead to an underestimate of coverage.

In summary, the challenges faced by many immunization programmes in understanding the target populations for their work is seemingly well recognized though not well documented, and it is the inadequacy of the available information, even with the aids of adjustments for differences over time, that constitutes the major weakness of target population estimates for many immunization programme managers. Sustained commitments and investment in timely, robust, and relevant data, including target population estimates, are critical to the future of immunization programmes worldwide $[11,12]$.

\section{CONFLICT OF INTEREST}

The authors confirm that this article content has no conflicts of interest.

\section{ACKNOWLEDGEMENTS}

None declared.

\section{DISCLAIMER}

The findings and views expressed herein are those of the authors alone and do not necessarily reflect those of their respective institutions.

\section{REFERENCES}

[1] United Nations Children's Fund (UNICEF). the 'rights' start to life: a statistical analysis of birth registration. New York: United Nations Children's Fund 2005. Available from www.unicef.org/protection/BirthReg10a_rev.pdf. [Accessed on 13 January, 2012].

[2] United Nations Children's Fund (UNICEF). ChildInfo: monitoring the situation of children and women, Birth Registration. Available from www.childinfo.org/birth_registration.html [Accessed on 13 January 2012].

[3] Bos E, Batson A. Using immunization coverage rates for monitoring health sector performance: measurement and interpretation. The World Bank: Washington, DC 2000.

[4] Siegel JS, Swanson DA. The methods and materials of demography. $2^{\text {nd }}$ ed. California, USA: Elsevier Academic Press 2004.

[5] United Nations Interagency Group on Child Mortality Estimation (IGME). www.childmortality.org/cmeMain.html; www.childinfo.org/files/Child_Mortality_Report_2011.pdf. [Accessed on 13 January, 2012].

[6] Burton AH, Monasch R, Lautenbach B, et al. WHO and UNICEF estimates of national infant immunization coverage: methods and processes. Bull World Health Organ 2009; 87: 535-41.

[7] Population data for surviving infants obtained from United Nations, department of Economic and Social Affairs, Population Division (2011). World Population Prospects: The 2010 Revision, CD-ROM ed.

[8] United Nations Development Policy and Analysis Division: The World Economic and Social Survey 2011. Available online at www.un.org/en/development/desa/policy/wess/

[9] MacDorman MF, Mathews TJ. Behind international rankings of infant mortality: How the United States compares with Europe. NCHS data brief, no 23. Hyattsville, MD: National Center for Health Statistics 2009.

[10] Brown DW, Feeney G, Burton AH. Raising awareness among immunization programme managers to the potential bias resulting from the application of fixed factors to obtain target population size estimates. Open Public Health J 2012; 5:15-18. Available online at http://benthamscience.com/ open/tophj/articles/V005/15TOPHJ.pdf

[11] Setel P, MacFarlane SB, Szreter S, et al. A scandal of invisibility: making everyone count by counting everyone. Lancet 2007; 370: 1569-77.

[12] Mahapatra P, Shibuya K, Lopez AD, et al. Civil registration systems and vital statistics: successes and missed opportunities. Lancet 2007; 370: 1653-63. 